\title{
Subject Index to Volume 9
}

Abstracts

XVII Canadian Congress of Neurological Sciences 265 (May)

Sixth National Scientific Workshop of the Muscular Dystrophy Association of Canada 349 (Aug)

Adrenoleukodystrophy

Adrenoleukodystrophy 449 (Nov)

Alcohol

Transient Choreiform Dyskinesias During Alcohol Withdrawal 89 (May)

Alzheimer's Disease

Hypothesis: Limbic Predilection in Alzheimer Dementia: Is Reactivated Herpes Virus Involved? 303 (Aug)

Amyloid Plaques

Spino-Cerebello-Cerebral Degeneration with Amyloid Plaques 439 (Nov)

Aneurysms

Clinical-Radiological Correlates in Intracerebral Hematomas due to Aneurysmal Rupture 409 (Nov)

Anticonvulsant

Cerebrospinal Fluid and Blood Thiamine Concentrations in Phenytoin-Treated Epileptics 37 (Feb)

Sodium Valproate in the Treatment of the Intractable Childhood Epileptic 307 (Aug)

Valproic Acid Producing a Reye-Like Syndrome 435 (Nov)

Ataxias, Inherited

Quebec Cooperative Study of Friedreich's Ataxia 91-263 (May)

Autism

Auditory Brainstem Response Abnormalities in Autistic Children 429 (Nov)

Benign Intracranial Hypertension Familial Benign Intracranial Hypertension and Depression 45 (Feb)

Birth Injury

Mechanisms of Brain Damage in Twins 339 (Aug)

Book Reviews

Book Reviews 64 (Feb)

Book Reviews 301 (May)

Book Reviews 371 (Aug)

Book Reviews 461 (Nov)

Catecholamines

Increased Plasma Catecholamines in Patients with Friedreich's Ataxia 195 (May)

Plasma Catecholamines in Friedreich's Ataxia: Assayed using High Performance Liquid Chromatography with Electrochemical Detection 205 (May)

Cerebellum

Heterotopic Growth of Dysplastic Cerebellum in Frontal Encephalocele in an Infant of a Diabetic Mother 31 (Feb)

Cerebrospinal Fluid

Cerebrospinal Fluid and Blood Thiamine Concentrations in Phenytoin-Treated Epileptics 37 (Feb)

Cerebrovascular Disease

Stroke in Coronary Bypass Surgery 21 (Feb)
Charcot-Marie-T ooth Disease

A Case of Charcot-Marie-Tooth Disease Mimicking Friedreich's Ataxia: Is There Any Association Between Friedreich's Ataxia and Charcot-Marie-Tooth Disease? 99 (May)

Congenital Malformations

Heterotopic Growth of Dysplastic Cerebellum in Frontal Encephalocele in an Infant of a Diabetic Mother 31 (Feb)

\section{Cushing's Disease}

Cushing's Disease: 50 Years Later 375 (Nov)

Cysticercosis

Childhood Cerebral Cysticercosis: Clinical Features and Computed Tomographic Findings in 89 Mexican Children 401 (Nov)

\section{Depression}

Familial Benign Intracranial Hypertension and Depression 45 (Feb)

\section{Diabetes Mellitus}

Multiple Sclerosis and Diabetes Mellitus: Further Evidence of a Relationship 415 (Nov)

The Effects of Intermittent Insulin Therapy on the Autonomic Neuropathy in the Streptozotocin Rat 79 (May)

Dopamine

Distribution of Dopamine in 35 Subregions of the Rat Caudate-Putamen: A High Performance Liquid Chromatography with Electrochemical Detection Analysis 421 (Nov)

Dystonia

Anticholinergics in Adult-Onset Focal Dystonia 313 (Aug)

\section{Electroretinogram}

Flash Electroretinogram Abnormalities in Patients with Clinically Definite Multiple Sclerosis 325 (Aug)

Encephalocele

Heterotopic Growth of Dysplastic Cerebellum in Frontal Encephalocele in an Infant of a Diabetic Mother 31 (Feb)

Epilepsy

Benign Familial Neonatal Convulsions 345 (Aug)

Cerebrospinal Fluid and Blood Thiamine Concentrations in Phenytoin-Treated Epileptics 37 (Feb)

Sodium Valproate in the Treatment of the Intractable Childhood Epileptic 307 (Aug)

Evoked Potential, Auditory

Auditory Brainstem Response Abnormalities in Autistic Children 429 (Nov)

Evoked Potential, Somatosensory

The Somatosensory Evoked Potential 65 (May)

Evoked Potential, Visual

Orientation-Specific Visual Evoked Potential Deficits in Multiple Sclerosis 331 (Aug)

Friedreich's Ataxia

Quebec Cooperative Study of Friedreich's Ataxia 91-263 (May)
Genetics

New Data on the Genetics of Parkinson's Disease 53 (Feb)

Hematoma, Intracerebral

Clinical-Radiological Correlates in Intracerebral Hematomas due to Aneurysmal Rupture 409 (Nov)

Hematoma, Epidural

Epidural Hematoma: Report of Seven Cases with Delayed Evolution of Symptoms 321 (Aug)

Hematopoiesis

Extramedullary Hematopoiesis Simulating Parasagittal Meningioma 49 (Feb)

Herpes Virus

Hypothesis: Limbic Predilection in Alzheimer Dementia: Is Reactivated Herpesvirus Involved? 303 (Aug)

Hypophysectomy, Transsphenoidal Cushing's Disease: 50 Years Later 375 (Nov)

Lecithin

Oral Lecithin and Linoleic Acid in Friedreich's Ataxia: I. Design of the Study, Material and Methods 151 (May)

Oral Lecithin and Linoleic Acid in Friedreich's Ataxia: II. Clinical Results 155 (May)

Oral Lecithin and Linoleic Acid in Friedreich's Ataxia: III. Biochemical Results 165 (May)

Linoleic Acid

Oral Lecithin and Linoleic Acid in Friedreich's Ataxia: I. Design of the Study, Material and Methods 151 (May)

Oral Lecithin and Linoleic Acid in Friedreich's Ataxia: II. Clinical Results 155 (May)

Oral Lecithin and Linoleic Acid in Friedreich's Ataxia: III. Biochemical Results 165 (May)

\section{Meningioma}

Extramedullary Hematopoiesis Simulating Parasagittal Meningioma 49 (Feb)

Meningitis

Le Meningite de Mollaret 41 (Feb)

Respiratory Arrest and Cervical Spinal Cord Infarction following Lumbar Puncture in Meningitis 443 (Nov)

Movement Disorders

Anticholinergics in Adult-Onset Focal Dystonia 313 (Aug)

Transient Choreiform Dyskinesias During Alcohol Withdrawal 89 (May)

\section{Multiple Sclerosis}

Flash Electroretinogram Abnormalities in Patients with Clinically Definite Multiple Sclerosis 325 (Aug)

Multiple Sclerosis and Diabetes Mellitus: Further Evidence of a Relationship 415 (Nov)

Orientation-Specific Visual Evoked Potential Deficits in Multiple Sclerosis 331 (Aug)

Plasma Exchange of Malignant Multiple Sclerosis 27 (Feb) 
Muscular Dystrophy

Sixth National Scientific Workshop of the Muscular Dystrophy Association of Canada 349 (Aug)

Myasthenia Gravis

Neonatal Myasthenia Gravis in the Infant of an Asymptomatic Thymectomized Mother 85 (May)

\section{Nerve Conduction}

Percutaneous Localization of Conduction Abnormalities in Human Entrapment Neuropathies 391 (Nov)

\section{Neuropathy}

Percutaneous Localization of Conduction Abnormalities in Human Entrapment Neuropathies 391 (Nov)

The Effects of Intermittent Insulin Therapy on the Autonomic Neuropathy in the Streptozotocin Rat 79 (May)

\section{Obituaries}

Dr. James K. Murray 61 (Feb)

Optic Nerve

The Symptomatology of Tumours of the Anterior Visual Pathways 381 (Nov)
Parkinson's Disease

New Data on the Genetics of Parkinson's Disease 53 (Feb)

Pituitary Adenoma Cushing's Disease: 50 Years Later 375 (Nov)

Plasmapheresis

Plasma Exchange of Malignant Multiple Sclerosis 27 (Feb)

Pseudotumour Cerebri

Familial Benign Intracranial Hypertension and Depression 45 (Feb)

Research, Clinical Neurosciences

Future Directions for Research in the Clinical Neurosciences: Policies, Strategy and Funding 3 (Feb)

Research in the Clinical Neurosciences 1 (Feb)

\section{Somatosensory System}

Parallel Processing of Cutaneous Information in the Somatosensory System of the Cat 9 (Feb)

The Somatosensory Evoked Potential 65 (May)
Spasticity

Pilot Study of Threonine Supplementation in Human Spasticity 141 (May)

Spinal Cord

Valproic Acid Producing a Reye-Like Syndrome 435 (Nov)

Spinocerebellar Degeneration

Quebec Cooperative Study of Friedreich's Ataxia 91-263 (May)

Spino-Cerebello-Cerebral Degeneration with Amyloid Plaques 439 (Nov)

Syndrome, Reye's

Valproic Acid Producing a Reye-Like Syndrome 435 (Nov)

Syndrome, Gerstmann-Straussler, Scheinker Spino-Cerebello-Cerebral Degeneration with Amyloid Plaques 439 (Nov)

Tumours

The Symptomatology of Tumours of the Anterior Visual Pathways 381 (Nov)

Visual Pathways

The Symptomatology of Tumours of the Anterior Visual Pathways 381 (Nov) 